\title{
Opposing white matter microstructure abnormalities in $22 q 11.2$ deletion and duplication carriers
}

\author{
Johanna Seitz-Holland $\mathbb{D}^{1 凶}$, Monica Lyons ${ }^{1}{ }^{1}$, Leila Kushan ${ }^{2}$, Amy Lin ${ }^{2}$, Julio E. Villalon-Reina ${ }^{2}$, Kang Ik Kevin Cho ${ }^{1}$, Fan Zhang $\mathbb{D}^{4}$,
} Tashrif Billah ${ }^{1}$, Sylvain Bouix (iD) ${ }^{1}$, Marek Kubicki (D) ${ }^{1,3,4}$, Carrie E. Bearden (iD) ${ }^{2,5}$ and Ofer Pasternak ${ }^{1,4}$

(c) The Author(s) 2021

Deletions and duplications at the $22 q 11.2$ locus are associated with significant neurodevelopmental and psychiatric morbidity. Previous diffusion-weighted magnetic resonance imaging (MRI) studies in 22q11.2 deletion carriers (22q-del) found nonspecific white matter (WM) abnormalities, characterized by higher fractional anisotropy. Here, utilizing novel imaging and processing methods that allow separation of signal contribution from different tissue properties, we investigate whether higher anisotropy is driven by (1) extracellular changes, (2) selective degeneration of secondary fibers, or (3) volumetric differences. We further, for the first time, investigate WM microstructure in 22q11.2 duplication carriers (22q-dup). Multi-shell diffusion-weighted images were acquired from 26 22q-del, 19 22q-dup, and 18 healthy individuals (HC). Images were fitted with the free-water model to estimate anisotropy following extracellular free-water elimination and with the novel BedpostX model to estimate fractional volumes of primary and secondary fiber populations. Outcome measures were compared between groups, with and without correction for WM and cerebrospinal fluid (CSF) volumes. In 22q-del, anisotropy following free-water elimination remained significantly higher compared with controls. BedpostX did not identify selective secondary fiber degeneration. Higher anisotropy diminished when correcting for the higher CSF and lower WM volumes. In contrast, 22q-dup had lower anisotropy and greater extracellular space than HC, not influenced by macrostructural volumes. Our findings demonstrate opposing effects of reciprocal 22q11.2 copynumber variation on WM, which may arise from distinct pathologies. In 22q-del, microstructural abnormalities may be secondary to enlarged CSF space and more densely packed WM. In 22q-dup, we see evidence for demyelination similar to what is commonly observed in neuropsychiatric disorders.

Translational Psychiatry (2021)11:580; https://doi.org/10.1038/s41398-021-01703-1

\section{INTRODUCTION}

Copy number variation (CNV) at the $22 q 11.2$ locus is associated with significant neurodevelopmental and psychiatric morbidity. Deletion of the 22q11.2 locus occurs in approximately one in 4000 live births [1]. Medical comorbidities commonly include cardiac defects [2], craniofacial anomalies [3], and immune dysregulation [4]. 22q11.2 deletion carriers (22q-del), furthermore, have elevated risk for autism spectrum disorder, attention deficit-hyperactivity disorder (ADHD), anxiety, intellectual disability, and up to $25 \%$ develop psychosis $[5,6]$. Duplication of the 22q11.2 locus is more common than the reciprocal deletion (estimated at one out of 700 live births) [7] but was more recently identified and is less wellcharacterized [8]. The 22q11.2 duplication is associated with incomplete penetrance and variable expressivity, even within the same family; associated features include hypotonia, facial dysmorphia, and developmental delay, although many 22q11.2 duplication carriers (22q-dup) have a normal or near-normal phenotype [9-11]. However, 22q-dup can suffer from a range of comorbidities that partially overlap those reported for $22 q$-del, including increased risk for autism, ADHD, and mild intellectual disability $[12,13]$.
In contrast, the 22q11.2 duplication is not associated with an elevated risk of developing psychosis [14], with some studies suggesting that the risk for psychosis in 22q-dup is lower than in the general population [15].

Given the importance of white matter (WM) microstructure for cognitive functioning [16], its disruption in numerous psychiatric and neurological disorders (including psychosis, mood disorders, obsessive-compulsive spectrum disorders, epilepsy, dementia, and Parkinson's disease) [17-24], and postmortem findings that implicate WM abnormalities in 22q-del [25, 26], there is a growing interest in characterizing the nature of WM microstructural anomalies in 22q-del [27, 28]. Studies to date have applied diffusion-weighted magnetic resonance imaging (MRI), which quantifies the directionality and magnitude of water diffusion in the brain, reflecting the geometrical properties of the environment in which water is diffusing $[29,30]$. The fractional anisotropy (FA) index, derived from the diffusion tensor imaging (DTI) model, is the most used index to quantify diffusion properties.

Lower FA has been implicated in multiple idiopathic psychiatric disorders (i.e., psychosis, depression, post-traumatic stress

\footnotetext{
${ }^{1}$ Department of Psychiatry, Brigham and Women's Hospital, Harvard Medical School, Boston 02115 MA, USA. ${ }^{2}$ Department of Psychiatry and Biobehavioral Sciences, Semel Institute for Neuroscience and Human Behavior, University of California at Los Angeles, Los Angeles 90095 CA, USA. ${ }^{3}$ Department of Psychiatry, Massachusetts General Hospital, Harvard Medical School, Boston 02114 MA, USA. ${ }^{4}$ Department of Radiology, Brigham and Women's Hospital, Harvard Medical School, Boston 02115 MA, USA. ${ }^{5}$ Department of Psychology, University of California at Los Angeles, Los Angeles 90095 CA, USA. ${ }^{\circledR}$ email: jseitz@bwh.harvard.edu
}

Received: 1 December 2020 Revised: 30 September 2021 Accepted: 15 October 2021

Published online: 10 November 2021 
disorder, and obsessive-compulsive spectrum disorders), as well as neurodegenerative disorders, and is often interpreted as a proxy for abnormal WM organization [31, 32]. However, while FA is a sensitive marker to distinguish groups, it is relatively non-specific and influenced by various cellular (e.g., demyelination, axonal degeneration, and cytoskeletal damage) and extracellular (e.g., atrophy and edema) pathologies [31, 33]. Thus, the neurobiological underpinnings of altered FA are unclear.

While there are some earlier studies that reported decreased FA in single WM tracts in 22q-del [27, 34, 35], most diffusionweighted MRI studies have reported a widespread increase of FA in 22q-del [28, 36-40]. Importantly a recent multi-site DTI study comparing $22 q-d e l$ with matched healthy individuals $(H C)$ confirmed the finding of higher FA in most tracts in 22q-del [41]. Studies investigating WM microstructure in 22q-dup, however, have not yet been conducted. Only one previous MRI study compared gray matter (GM) structure in reciprocal $22 q 11.2$ CNVs. The authors discovered global opposing effects of gene dosage on cortical thickness and surface area, involving widespread reductions in cortical surface area in 22q-del and increases in $22 q$-dup relative to controls, with the opposite pattern for cortical thickness [42].

In the present study, we compared diffusion MRI data between reciprocal 22q11.2 CNVs and tested whether opposing effects of gene dosage, similar to the effects reported for GM, also occur in WM. To determine the potential mechanisms that might drive FA alterations, we applied advanced dMRI analysis approaches which allowed considering: (1) extracellular and cellular changes, as quantified by free-water (FW) imaging [43]; (2) the role of selective degeneration of crossing fibers, as quantified by the BedpostX model [44, 45]; and (3) the interaction between microstructural diffusion measurements and macrostructural volume changes.

\section{METHODS \\ Participants}

The sample consisted of 26 22q-del, 19 22q-dup, and $18 \mathrm{HC}$ that passed visual quality control. Patients have been recruited from either (1) Clinical Genetics, Allergy/Immunology, or Craniofacial Clinics from medical centers in the Southern California area, or (2) through local support groups and websites. HC were recruited from the community via webbased advertisements and local schools, pediatric clinics, and community sites. Exclusion criteria for all participants were significant neurological or medical conditions (unrelated to 22q11.2 deletion/duplication), history of head injury with loss of consciousness, insufficient fluency in English, and/or substance abuse or dependency within the past six months. Further exclusion criteria for $\mathrm{HC}$ were current or past major mental disorders (except for ADHD or past episodes of depression) and/or intellectual disability (IQ below 70). HC were screened for mental disorders via the Structured Clinical Interview for the Diagnostic and Statistical Manual of Mental Disorders Version 4 [46] or Computerized Diagnostic Interview for Children.

All participants or parents, if participants were under the age of 18 , provided written informed consent, and the University of California at Los Angeles Institutional Review Board approved all study procedures.

\section{Cognitive and clinical assessment}

Participants underwent a comprehensive clinical and cognitive test battery administered by trained clinical psychology students and supervised by PhD-level clinicians.

Given the previously reported high rates of neuropsychiatric disorders in 22q-del and 22q-dup [11, 12, 47-49], we collected extensive clinical and cognitive data for the present study. We utilized the Structured Clinical Interview for DSM V (SCID-I) and the Brief Psychiatric Rating ScaleExpanded Version (BPRS) [50-52] to assess neuropsychiatric diagnosis and symptoms. In addition, the Structured Interview for Psychosis-Risk Syndromes (SIPS) [53] was administered to assess for psychotic and prodromal (i.e., psychosis-risk) symptoms. Previous research has suggested that many individuals with the $22 \mathrm{q}$-del present with prodromal symptoms [54] and up to $25 \%$ develop psychosis [55, 56, 6]. 22q-dup, on the other hand, does not have an elevated risk for psychosis [14], and in fact, there is some evidence suggesting a lower risk for psychosis than in the general population [15].

Furthermore, we were interested in characterizing global and cognitive functioning. Functional status was, therefore, determined by the Global Functioning Scales (GAF) [57]. In addition, the Wechsler Abbreviation Scale for Intelligence-2 Vocabulary (VOC) and Matrix Reasoning (Matrix) subtests and the Wide Range Achievement Test 4 (WRAT) Reading subtest were used to assessing intelligent quotient (IQ) and reading ability, respectively $[58,59]$.

\section{Image acquisition and preprocessing}

Images were acquired on a $3 \mathrm{~T}$ whole-body scanner (Siemens Magnetom Prisma) with a 32-channel head coil. Scanning protocols were derived from the Lifespan Human Connectome Project (HCP) study, including multi-shell diffusion-weighted images that were acquired with the following protocol: $\mathrm{AP}$ and $\mathrm{PA}$ sequence each with voxel size $=2.0 \times 2.0 \times 2.0 \mathrm{~mm}$, TR $=$ $8000 \mathrm{~ms}, \mathrm{TE}=66 \mathrm{~ms}$, flip angle $=90^{\circ}, \mathrm{FOV}=208 \mathrm{~mm}$, slice thickness $=$ $2.00 \mathrm{~mm}$, slices $=72$, and 108 volumes, including 46 gradient directions with $b=1500 \mathrm{~s} / \mathrm{mm}^{2}, 46$ gradient directions with $b=3000 \mathrm{~s} / \mathrm{mm}^{2}, 3$ gradient directions with $b=200 \mathrm{~s} / \mathrm{mm}^{2}, 6$ gradient directions with $b=500 \mathrm{~s} / \mathrm{mm}^{2}$, and 7 volumes with $b=0 \mathrm{~s} / \mathrm{mm}^{2}$.

All data underwent a visual quality check for movement artifacts, echoplanar imaging (EPI) distortions, and structural abnormalities by investigators blinded to group allocation. We excluded individuals who did not have complete data for the AP and PA sequence and individuals who presented with severe motion artifacts (excluding a total of 25 individuals: $5 \mathrm{HC}, 12$ 22q-del, and $822 \mathrm{q}$-dup). We preprocessed the remaining images utilizing the HCP Minimal Preprocessing Pipeline v4.0.0 [60]. First, we applied intensity normalization of the mean b0 image across the diffusion series and utilized b0 pairs to estimate and correct EPI distortion, using Topup, FSL [61]. Next, diffusion images were corrected for eddy-current induced field inhomogeneities, head motion, and gradient nonlinearity utilizing FSL. Last, we used structural images to mask diffusion images [62].

\section{Image analyses}

FA Analyses. We first investigated group differences in FA to determine the comparability of our findings to previous findings of increased FA in 22q-del. From the preprocessed diffusion-weighted images, we, therefore, computed diffusion tensors utilizing a least-squares fit and calculated FA maps from these tensors [63].

Cellular and extracellular analyses. To identify if the FA abnormalities originate from the cellular or extracellular domains, we fitted a twocompartment FW model to the multi-shell diffusion data using a regularized non-linear fit [43]. One compartment models signals from water molecules that diffuse unhindered in the extracellular space using an isotropic diffusion tensor with a fixed diffusivity of FW in body temperature. The fractional volume of this FW compartment is identified as FW. Previous studies have demonstrated that an increase of FW indicates extracellular pathological processes such as atrophy, edema, and neuroinflammation [64]. The second compartment models hindered and restricted diffusion of water molecules in the vicinity of the cellular space using a diffusion tensor, from which $F A$ of the tissue $\left(F A_{T}\right)$ is derived $[43,64,65] . \mathrm{FA}_{\mathrm{T}}$ represents anisotropy following the elimination of $\mathrm{FW}$ contribution, and therefore $\mathrm{FA}_{\mathrm{T}}$ more specifically reflects changes in the WM tissue (such as changes in myelination and axonal membrane health) than FA $[43,66]$. FW imaging studies in healthy aging and in several disorders (including psychosis [67, 68], mood disorders [69, 70], eating disorders [71], traumatic brain injury [72], dementia [73, 74], and Parkinson's disease $[75,76])$ have demonstrated the importance of separating cellular and extracellular WM abnormalities [77].

Identification of secondary degeneration. We used the crossing fiber BedpostX model [44] to obtain information about the complex fiber architecture at each voxel. Previous studies have suggested that increased FA could result from selective degeneration of secondary fibers in regions with crossing fibers $[78,79]$. This model considers three fiber populations with distinct orientations and an additional isotropic compartment. The model parameters are estimated in each voxel using a Bayesian framework and include the volume fractions for the primary (F1), secondary (F2), and tertiary (F3) fiber populations, as well as the fractional volume of the isotropic compartment $\left(F_{\text {iso }}\right)$. Degeneration of secondary fibers is expected to be indicated by a decrease of F2 but not F1. Previous studies have demonstrated that this selective degeneration of secondary fibers yields 


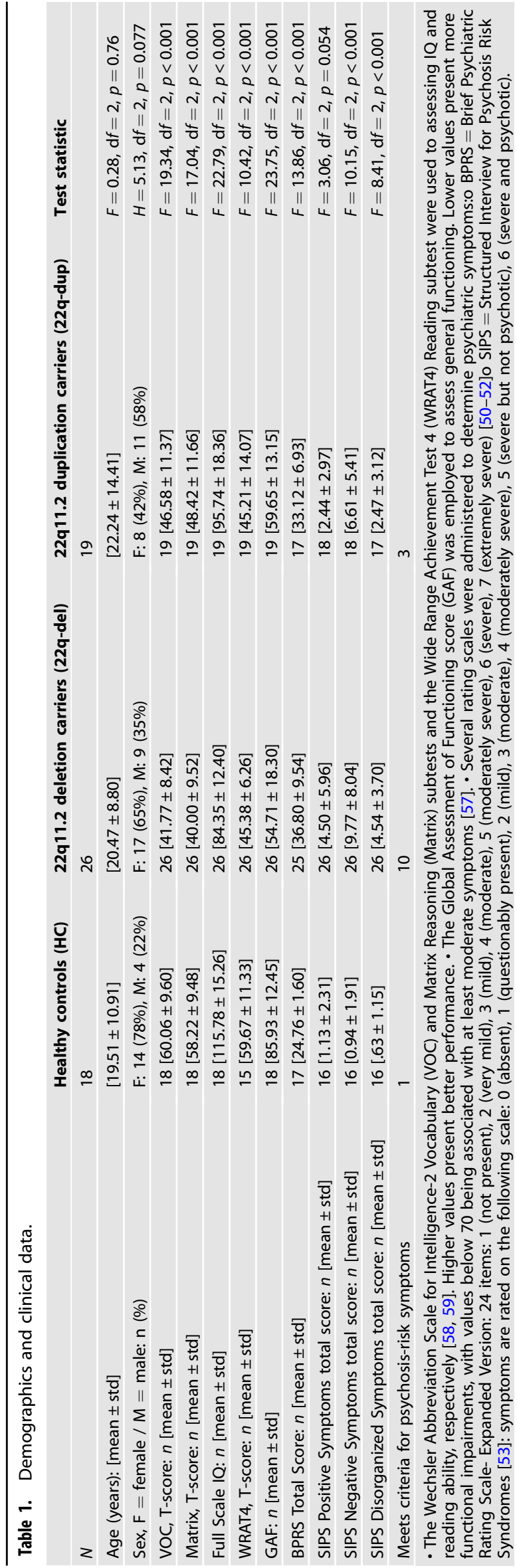

less partial volume in crossing fibers areas, resulting in an increase of FA values [78].

Tract-based spatial statistics. To facilitate group comparisons, we employed the tract-based spatial statistics (TBSS) pipeline [80-82] by nonlinear registration of each subject's FA image to the FA target image and skeleton that was created by the Enhanced Neuroimaging Genetics by Meta-Analysis (ENIGMA) DTI Working Group. We used an in-house script (https://pnlbwh.github.io/TBSS/TUTORIAL.html) that performed all registrations using the Advanced Normalization Tools (ANTs) toolbox [83, 84]. FA, $\mathrm{FA}_{\mathrm{T}}$, and $\mathrm{FW}$ maps were projected onto the ENIGMA skeleton [85] to perform statistical analyses.

On the output of the BedpostX model, we applied FSL's tbss_x script. This script provides intra- and inter-subject reassignment of fiber fractions, thus ensuring that a fiber population in one subject corresponds to the same fiber population across all individuals [45]. We projected F1, F2, and $F_{\text {iso }}$ maps onto the skeleton provided by ENIGMA to perform statistical analyses. (F3 was not included in the analyses due to its inherently low and inconsistent values [86]).

Accounting for macrostructural volumetric measures. To test if macrostructural volume measures interact with the microstructural measures, we utilized a novel segmentation algorithm [87] that allowed for the segmentation of the diffusion image into CSF, WM, GM, and total intracranial volume (ICV, sum of $\mathrm{GM}+\mathrm{WM}+\mathrm{CSF})$. Following this, we calculated normalized measurements by dividing CSF, WM, and GM volumes by ICV. We used these normalized volumes for all statistical analyses.

\section{Statistical analyses}

For all TBSS analyses, non-parametric voxel-wise permutation tests for each voxel on the WM skeleton were performed in FSL's Randomize [81]. We tested data against a null distribution generated with 5000 permutations for each contrast using threshold-free cluster enhancement [82] and family wise error correction at a significance level of $p<0.05$ and included age, sex, and motion as covariates.

For each of the maps $\left(F A, F A_{T}, F W, F 1, F 2\right.$, and $F_{\text {iso }}$, we conducted voxelwise $F$-tests and post hoc $t$-tests to compare the three groups (22q-del, 22q-dup, and $\mathrm{HC}$ ).

We performed all subsequent analyses using SPSS Version 26 and GraphPad Prism 8.2.0. First, we correlated $F A_{T}$ and $F W$ values with IQ $[58,59]$, global functioning [57], and the positive and negative symptom scales measured with the Structured Interview for Prodromal Syndromes (SIPS) [53] for 22q-del and 22q-dup separately. A $p$-value $<.013$ was considered significant (Bonferroni corrected for four tests).

Next, to investigate group differences in macrostructural volume, we carried out four ANCOVAs with ICV, CSF, WM, or GM volume as the independent variable, group as the dependent variable (22q-del, 22qdup, and HC), and age, sex, and motion as covariates. A $p$-value $<0.013$ was considered significant (Bonferroni corrected for four tests). In the case of a significant group effect in the ANCOVA, we then calculated post hoc $t$-tests between the single groups ( $p$-values were again Bonferroni corrected).

Last, we included the volumes that demonstrated group differences in our previous analyses to identify the effect of macrostructural volume on microstructure. We, therefore, repeated the voxel-wise $F$-test and post-hoc t-tests comparing $F A_{T}, F W, F 1, F 2$, and $F_{\text {iso, }}$ between groups with the volumes that were different between groups as an additional covariate.

\section{RESULTS \\ Demographics}

Demographic information is shown in Table 1. The three groups did not differ in terms of age or sex. 22q-del and 22q-dup had poorer cognitive performance and general functioning than $\mathrm{HC}$, with impairments being more pronounced for 22q-del. In addition, both groups reported more psychiatric symptoms than HC. As displayed in Table 2, the most frequent DSM-V diagnoses in $22 \mathrm{q}$ del and 22q-dup were anxiety disorders, autism spectrum disorder, ADHD, and mood disorders. As described previously $[6,55,56], 22 q-d e l$ reported more prodromal psychotic symptoms than $22 q$-dup and HC. However, only four 22q-del were diagnosed with overt psychotic disorder. 
Table 2. Psychiatric diagnoses in CNV carriers*.

\begin{tabular}{|lll|}
\hline & $\mathbf{2 2 q 1 1 . 2}$ deletion carriers (22q-del) (\%) & 22q11.2 duplication carriers (22q-dup) (\%) \\
\hline Substance-related disorders & 3.85 & 0 \\
\hline Schizophrenia and other psychotic disorders & 11.54 & 0 \\
\hline Mood disorders & 26.92 & 26.32 \\
\hline Anxiety disorders & 61.54 & 42.11 \\
\hline Obsessive-compulsive spectrum disorder & 7.69 & 0 \\
\hline Eating disorders & 3.85 & 0 \\
\hline Autism Spectrum Disorder & 34.62 & 31.58 \\
\hline Attention deficit hyperactivity disorder & 46.15 & 52.63 \\
\hline
\end{tabular}

${ }^{*}$ Obtained from the Structural Clinical Interview for DSM V (SCID-I)

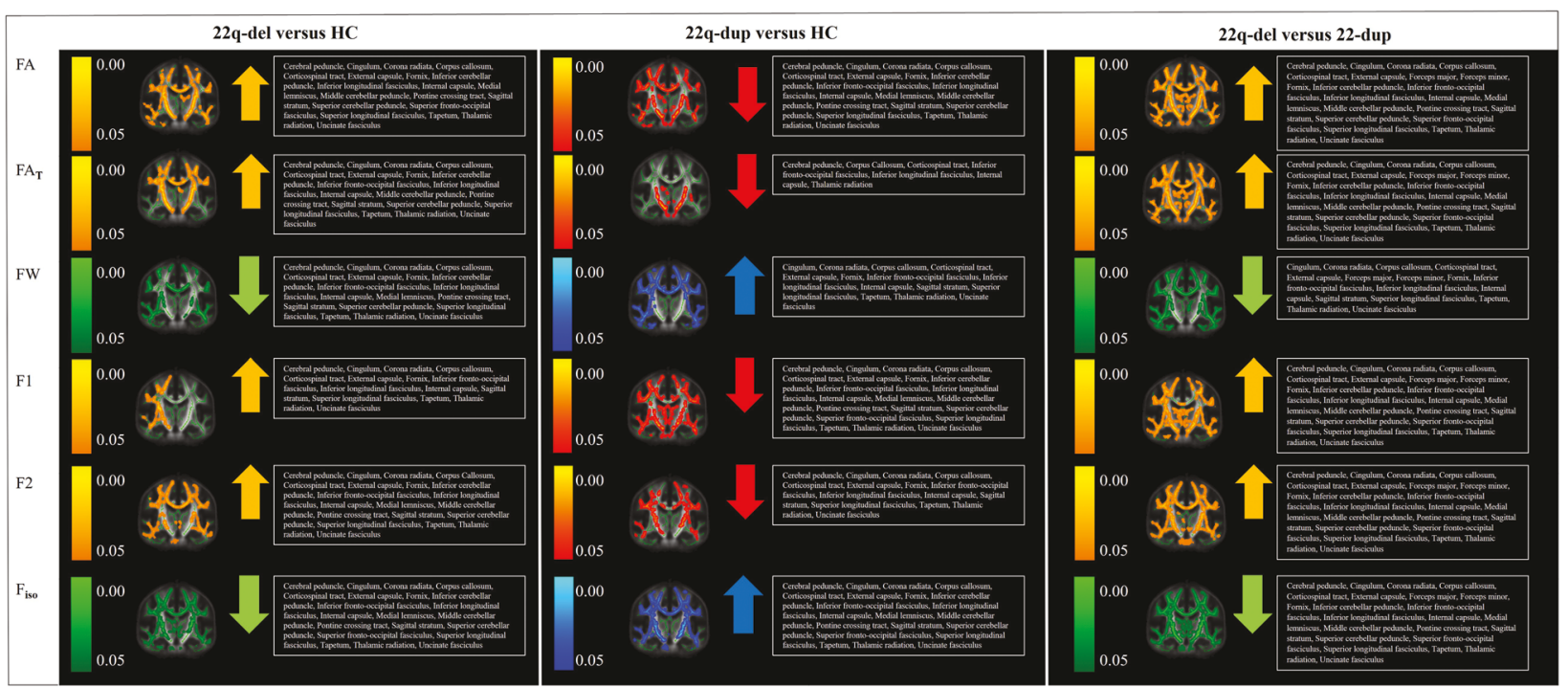

Fig. 1 Group comparisons between 22q11.2 deletion carriers (22q-del) and healthy controls (HC) (left), 22q11.2 duplication carriers (22q-dup) and HC (middle), and 22q-del and 22q-dup. Figure 1 displays the results from Tract-Based Spatial Statistics and Randomize [81, 82]. The white matter skeleton (thresholded at fractional anisotropy $(F A)>0.25$ ) is shown in green on top of the average image of all registered FA images. Voxels that demonstrated significant group differences are thickened to increase visibility. The 22q-del group compared with the HC group (left) showed higher fractional anisotropy ( $F A$; significant regions highlighted in orange), which remained higher in $F A$ of the tissue $\left(F A_{T}\right)$ values (in orange) after accounting for the effect of extracellular free-water (FW). 22q-del also showed lower FW values than HC (in green) and higher fractions of both the primary (F1) and secondary (F2) fiber populations (in orange), and lower fractional volume of the isotropic compartment $F_{\text {iso }}$ (in green). In contrast, 22q-dup compared with HC (middle) presented with lower FA values (in red), as well as lower FA (in red), higher FW values (in blue), lower fractions of the primary and secondary fiber populations ( $F 1, F 2$, in red) and higher $F_{\text {iso }}$ (in blue). A direct comparison between 22q-del and 22q-dup is provided on the right, showing widespread group differences. 22q-del displayed higher FA/FA $/ F 1 / F 2$ (in orange) and lower $F W / F_{\text {iso }}$ (in green). Please note that TBSS provides a voxel-wise rather than tract-specific output. The voxels that demonstrate group differences are widespread and located in most of the main fiber tracts. For a tract-by-tract comparison of FA $\mathrm{A}_{T}$ and FW between groups, please see Supplementary Table 1.

\section{FA analyses}

An $F$-test revealed a significant group effect on FA in $54 \%$ of the WM skeleton. Post hoc $t$ tests demonstrated that 22q-del had significantly higher FA than HC in $41 \%$ of the WM skeleton, and 22 -dup had significantly lower FA than HC in $37 \%$ of the WM skeleton. Further, 22q-del displayed significantly higher FA than $22 q$-dup in $66 \%$ of the WM skeleton (Fig. 1).

\section{Cellular and extracellular analyses}

F-tests comparing the output of the FW imaging measures revealed significant group effects on $\mathrm{FA}_{\mathrm{T}}$ in $45 \%$ of the WM skeleton and on FW in $72 \%$ of the WM skeleton. Post hoc $t$ tests revealed that relative to $\mathrm{HC}, 22 \mathrm{q}$-del had higher $\mathrm{FA}_{\mathrm{T}}$ (in $24 \%$ of the WM skeleton) and lower FW (in 49\% of the WM skeleton). 22q-dup presented with the opposite pattern: lower $\mathrm{FA}_{\mathrm{T}}$ (in $8 \%$ of the WM skeleton) and higher FW (in 59\% of the WM skeleton) than $\mathrm{HC}$. In addition, 22q-del demonstrated higher $\mathrm{FA}_{\mathrm{T}}$ (54\% of the WM skeleton) and lower FW (76\% of the WM skeleton) than 22q-dup (Fig. 1, Table 3).
We did not observe a significant correlation between $\mathrm{FA}_{\mathrm{T}}$ or $\mathrm{FW}$ and IQ [58,59], global functioning [57], nor the SIPS positive and negative symptom scales in either $22 \mathrm{q}$-del or $22 \mathrm{q}$-dup carriers.

\section{Complex fiber architecture and the identification of selective degeneration}

The F-tests comparing the BedpostX fractional volume measures between groups showed a significant group effect on the fraction of both primary (F1 in 36\% of the WM skeleton) and secondary (F2 in $40 \%$ of the WM skeleton) fibers, as well as the partial volume fraction of the isotropic compartment $\left(F_{\text {iso }}\right.$ in $66 \%$ of the WM skeleton). Post-hoc t-tests revealed that 22q-del had higher primary ( $\mathrm{F} 1$ in $8 \%$ of the WM skeleton) and secondary (F2 in $28 \%$ of the WM skeleton) fiber fractions and a lower isotropic fraction ( $\mathrm{F}_{\text {iso }}$ in $49 \%$ of the WM skeleton) than $\mathrm{HC}$, which is not consistent with a pattern of degeneration of secondary fibers $[78,79]$. Similar to the FA results, 22 -dup presented with the opposite pattern: lower primary ( $26 \%$ of the WM skeleton) and 
Table 3. $\mathrm{FA}_{\mathrm{T}}$ and $\mathrm{FW}$ group comparisons *.

\section{Covariates: age, sex, motion}

$F A_{T}$

F-test

22q-del versus $\mathrm{HC}$

22q-dup versus HC

22q-del versus $22 q$-dup

FW

F-test

22q-del versus $\mathrm{HC}$

22q-dup versus $\mathrm{HC}$

$22 q$-del versus $22 q$-dup

\section{$45 \%$ of the WM skeleton}

22q-del < HC: -

22q-dup < HC: $8 \%$

22q-del > 22q-dup: $54 \%$

22q-del < 22q-dup: -

$72 \%$ of the WM skeleton

22q-del > HC: -

22q-del < HC: $49 \%$

22q-del > 22q-dup: -

22q-del < 22q-dup: $76 \%$ 22q-del > HC: $24 \%$

22q-dup > HC: -

22q-dup > HC: $59 \%$

22q-dup < HC: -
Covariates: age, sex, motion, CSF volume

$24 \%$ of the WM skeleton

22q-del > HC: -

22q-del < HC: -

22q-dup > HC: -

22q-dup < HC: $23 \%$

22q-del > 22q-dup: $40 \%$

22q-del < 22q-dup: -

\section{$63 \%$ of the WM skeleton}

22q-del > HC: -

$22 \mathrm{q}-$ del < HC: $3 \%$

22q-dup > HC: $60 \%$

22q-dup < HC: -

22q-del > 22q-dup: -

22q-del < 22q-dup: $69 \%$
Covariates: age, sex, motion, WM volume

$\mathbf{2 7 \%}$ of the WM skeleton

22q-del > HC: $1,5 \%$

22q-del < HC: -

22q-dup > HC: -

22q-dup < HC: $7 \%$

22q-del > 22q-dup: $35 \%$

22q-del < 22q-dup: -

$\mathbf{5 5 \%}$ of the WM skeleton

22q-del > HC: -

22q-del < HC: -

22q-dup > HC: $60 \%$

22q-dup < HC: -

22q-del > 22q-dup: -

22q-del < 22q-dup: $60 \%$

CSF cerebrospinal fluid, $F A_{T}$ fractional anisotropy of cellular tissue, $F W$ extracellular free-water, $H C$ healthy controls, WM white matter, 22q-del 22q11.2 deletion carriers, 22q-dup 22q11.2 duplication carriers.

*All group comparisons were conducted utilizing non-parametric voxel-wise permutation tests for each voxel on the WM skeleton in FSL's Randomize [81]. We tested data against a null distribution generated with 5000 permutations for each contrast using threshold-free cluster enhancement [82] and family wise error correction at a significance level of $p<0.05$.
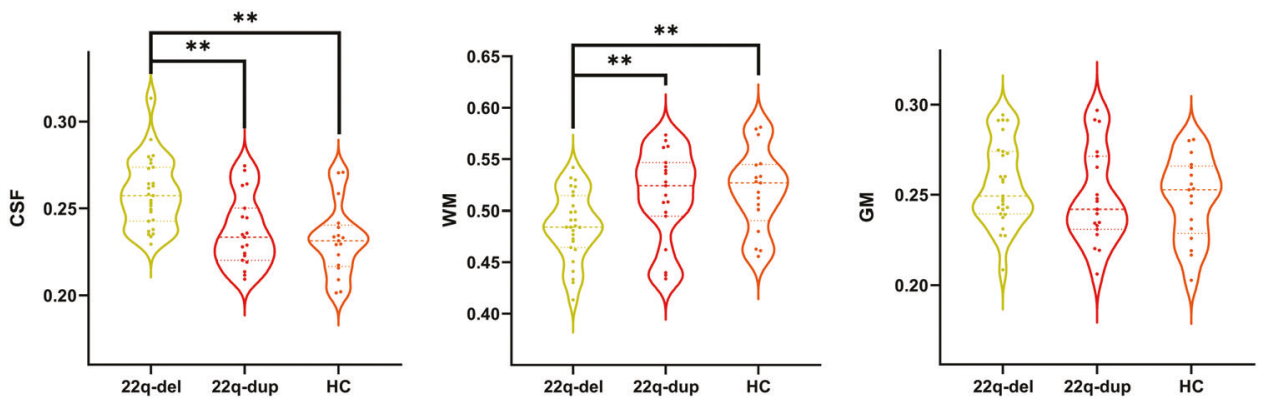

Fig. 2 Macrostructural volumetric measures across groups. Group comparison for relative cerebrospinal fluid volume (CSF), relative white matter (WM) volume, and relative gray matter (GM) volume between 22q11.2 deletion carriers (22q-del), 22q11.2 duplication carriers (22qdup), and healthy controls (HC). 22q-del had significantly higher CSF volume and lower WM volume relative to $22 \mathrm{q}$-dup and HC. GM volume did not significantly differ between groups.

secondary ( $21 \%$ of the WM skeleton) fiber fractions than $\mathrm{HC}$ and a higher isotropic fraction (in $48 \%$ of the WM skeleton) than HC (Fig. 1), which also is not consistent with a pattern of degeneration of secondary fibers $[78,79]$. For group comparisons between $22 q-$ del and 22q-dup, please see Table 3.

\section{Comparison of macrostructural volumetric measures}

We did not observe a significant overall group effect on ICV $(F=$ $3.75, \mathrm{~d} f 1=2, \mathrm{~d} f 2=58, p=0.029)$ or $\mathrm{GM}$ volume $(F=2.07, \mathrm{~d} f 1=2$, $\mathrm{d} f 2=58, p=0.14)$. However, there was a significant group effect on WM volume $(F=17.26, \mathrm{~d} f 1=2, \mathrm{~d} f 2=58, p<0.001)$ and CSF volume $(F=28.54, \mathrm{~d} f 1=2, \mathrm{~d} f 2=58, p<0.001)$. Post hoc $t$-tests revealed that $22 \mathrm{q}$-del had lower WM volume than both $22 \mathrm{q}$-dup $(p=0.062)$ and HC $(p=0.050)$. In addition, 22q-del had higher CSF volume than both $22 q-d u p(p=0.002)$ and HC $(p<0.001)$ (Fig. 2).

\section{Accounting for macrostructural volumetric measures}

Given the group effect on CSF and WM volume, we conducted two additional analyses of the voxel-wise $F$ - and $t$ tests comparing $F A_{T}, F W, F 1, F 2$, and $F_{i s o}$ : once controlling for CSF, and once controlling for WM volume. This was done to test whether the microstructural impairments reported above could be accounted for by volumetric differences between groups. Indeed, when correcting previous analyses for WM volume, differences between 22q-del and $\mathrm{HC}$ in FW and $\mathrm{F} 1$ were no longer significant, and differences in $\mathrm{FA}_{\mathrm{T}}, \mathrm{F} 2$, and $\mathrm{F}_{\text {iso }}$ were more localized (Table 3, Table 4, and Fig. 3). Similarly, when adjusting previous analyses for CSF volume, differences between $22 \mathrm{q}$-del and $H C$ in $F A_{T}$ and $F 1$ were no longer significant, and differences in FW, F2, and $F_{\text {iso }}$ were more localized (Table 3, Table 4, and Fig. 3). Notably, group differences between 22q-dup and HC remained stable after adding CSF or WM volume as covariates (Table 3, Table 4, and Fig. 3).

\section{DISCUSSION}

The present study provides novel information regarding WM abnormalities in 22q11.2 CNV carriers. First, using new multi-shell diffusion sequences, we confirmed previous findings of abnormally high FA in 22q-del. Further analyses demonstrated that the 
Table 4. F1, F2, and $F_{\text {iso }}$ group comparisons*.

\section{Covariates: age, sex, motion}

F1

$F$-test

22q-del versus $\mathrm{HC}$

22q-dup versus $\mathrm{HC}$

22q-del versus 22q-dup

F2

$F$-test

22q-del versus $\mathrm{HC}$

22q-dup versus $\mathrm{HC}$

22q-del versus $22 q-d u p$

$F_{\text {iso }}$

$F$-test

22q-del versus $\mathrm{HC}$

22q-dup versus $\mathrm{HC}$

22q-del versus $22 q$-dup 22q-del > HC: $8 \%$

$22 \mathrm{q}-\mathrm{del}<\mathrm{HC}:-$

22q-dup > HC: -

22q-dup < HC: $26 \%$

22q-del > 22q-dup: $43 \%$

22q-del < 22q-dup: -

$\mathbf{4 0 \%}$ of the WM skeleton

22q-del > HC: $28 \%$

22q-del < HC: -

22q-dup > HC: -

22q-dup < HC: $21 \%$

22q-del > 22q-dup: $46 \%$

22q-del < 22q-dup: -

$66 \%$ of the WM skeleton

22q-del > HC: -

22q-del < HC: $49 \%$

22q-dup > HC: $48 \%$

22q-dup < HC: -

22q-del > 22q-dup: -

22q-del < 22q-dup: $72 \%$
$36 \%$ of the WM skeleton
Covariates: age, sex, motion CSF volume

\section{$32 \%$ of the WM skeleton}

22q-del > HC: -

22q-del < HC: -

22q-dup > HC: -

22q-dup < HC: $29 \%$

22q-del > 22q-dup: 31\%

22q-del < 22q-dup: -

$\mathbf{2 6 \%}$ of the WM skeleton

22q-del > HC: $12 \%$

22q-del < HC: -

22q-dup > HC: -

22q-dup < HC: $21 \%$

22q-del > 22q-dup: 34\%

22q-del < 22q-dup: -

\section{$55 \%$ of the WM skeleton}

22q-del > HC: -

$22 q-$ del $<$ HC: $18 \%$

22q-dup > HC: $52 \%$

22q-dup < HC: -

22q-del > 22q-dup: -

22q-del < 22q-dup: 64\%
Covariates: age, sex, motion, WM volume

\author{
$14 \%$ of the WM skeleton \\ 22q-del > HC: - \\ 22q-del < HC: - \\ 22q-dup > HC: - \\ 22q-dup < HC: $21 \%$ \\ 22q-del > 22q-dup: $21 \%$ \\ 22q-del < 22q-dup: -
}

CSF cerebrospinal fluid, F1 fractional volume of primary fiber population, F2 fractional volume of secondary fiber population, $F_{i s o}$ fractional volume of the isotropic compartment, $H C$ healthy controls, WM white matter, 22q-del 22q11.2 deletion carriers, 22q-dup 22q11.2 duplication carriers.

*All group comparisons were conducted utilizing non-parametric voxel-wise permutation tests for each voxel on the WM skeleton in FSL's Randomize [81]. We tested data against a null distribution generated with 5000 permutations for each contrast using threshold-free cluster enhancement [82] and family wise error correction at a significance level of $p<0.05$.

observed higher FA remains following the elimination of extracellular FW and cannot be attributed to selective degeneration of secondary fibers. However, higher FA, which was accompanied by higher primary and secondary fiber fractions and lower extracellular space, was associated with enlarged CSF and smaller WM volumes in 22q-del. The finding of reduced WM volume and reduced extracellular space within the WM suggests that the WM in 22q-del may be abnormally densely packed.

Further, we found that 22q-del presents with distinct WM microstructural abnormalities compared to those with 22q-dup. In 22q-dup, we found significantly lower FA and lower primary and secondary fiber fractions, as well as higher extracellular space, relative to controls. This pattern is similar to what has been observed for many behaviorally defined neuropsychiatric disorders, such as psychosis, mood disorders, obsessive-compulsive disorder, and dementia [88-93]. In contrast to 22q-del, the microstructural changes were not associated with volumetric changes, suggesting that distinct pathologies may underlie WM pathology in reciprocal 22q11.2 CNVs (Fig. 4).

\section{WM architecture in $\mathbf{2 2 q - d e l}$}

We observed widespread higher FA in 22q-del than HCs, affecting most of the main WM tracts, and no regions with lower FA in $22 q$ del. Previous studies examining FA in 22q-del described inconsistent results. Some smaller studies suggested lower FA in localized tracts in 22q-del compared to HC [94, 27, 34, 95, 35]. Howeveraligning with our findings-prior studies found widespread increased FA [28, 36, 37], along with decreased extracellular space [96]. Notably, the most extensive multi-site study to date [41] also demonstrated lower diffusivity in 22q-del and higher FA in most fiber tracts. As a possible explanation for the inconsistencies, the authors speculated that FA findings may be age-dependent $[39,40]$.

To better understand the finding of higher FA in our sample, we examined if it is driven by (1) extracellular changes, (2) selective degeneration of secondary fibers, or (3) macrostructural volumetric effects. Utilizing the $\mathrm{FW}$ imaging approach $[43,64]$ to control for extracellular changes, widespread higher $F A_{T}$ was still observed in 22q-del, suggesting that extracellular changes alone did not explain the increase in FA. We also found lower FW, indicating a smaller extracellular space within WM in 22q-del than HC. Using the BedpostX method, which allows us to differentiate the primary and secondary fiber populations in one voxel, we found a higher proportion of both primary and secondary fibers in 22q-del compared to HC. This pattern is not consistent with selective degeneration of crossing fibers, which was found to cause higher FA in regions with crossing fibers in healthy aging and Alzheimer's Disease populations [78, 79]. Instead, the BedpostX results in $22 \mathrm{q}$-del aligned with the FW imaging results and showed a higher proportion of cellular volume at the expense of smaller extracellular volume at each voxel. In addition, we observed lower overall WM volumes and increased CSF volumes in $22 \mathrm{q}$-del relative to HC. Importantly, when adjusting our statistical analyses for these macrostructural group differences, the changes in $F A$ and $F A_{T}$ between $22 q$-del and $\mathrm{HC}$ were no longer significant. 


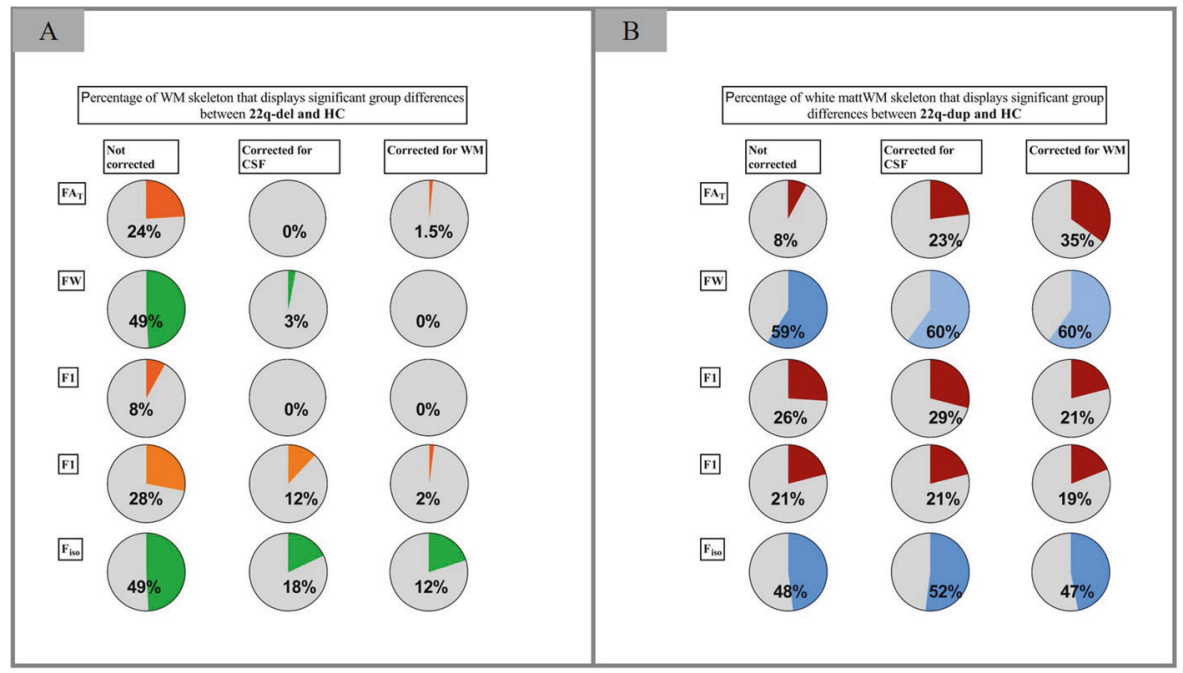

Fig. 3 The effect of controlling for macrostructural volume on the percentage of white matter (WM) skeleton that displays significant group differences. Comparing 22q11.2 deletion carriers (22q-del) and healthy controls (HC) (Panel A) shows that without controlling for macrostructural volume (left column), 22q-del displays significant differences in all microstructural parameters (FAt, FW, F1, F2, $F_{\text {iso }}$ ). When controlling for relative cerebrospinal fluid (CSF) volume (middle column) or relative WM volume (right column), these group differences disappear or diminish. Comparing 22q11.2 duplication carriers (22q-dup) and HC (Panel B) shows that the percentage of skeleton voxels with significant findings (left column) remain similar when controlling for CSF volume (middle row) or WM volume (right row).

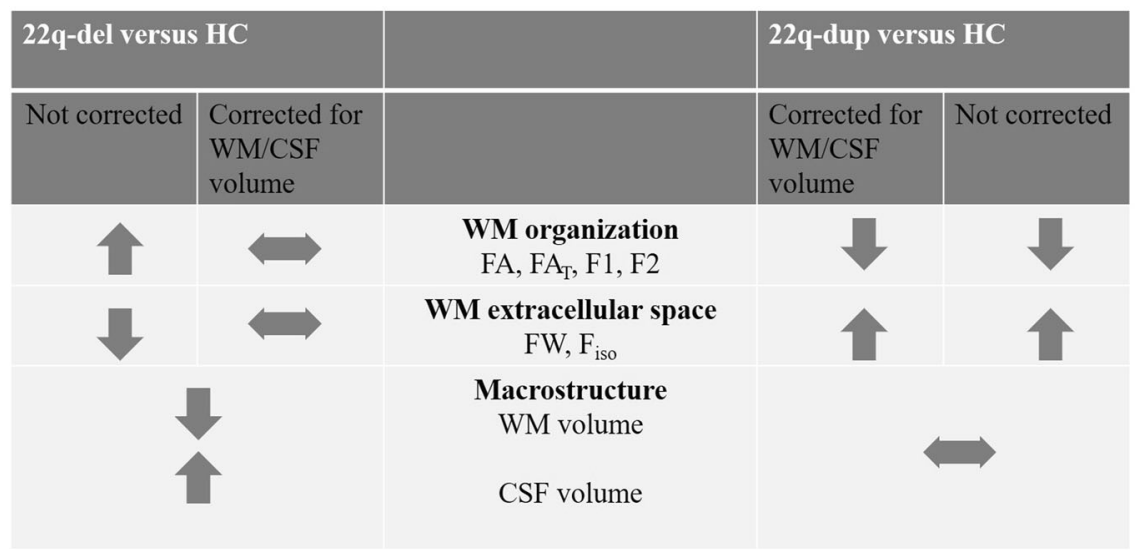

Fig. 4 Summary of overall results. When not correcting for macroscopic volumes, 22q11.2 deletion (22q-del) and 22q11.2 duplication carriers (22q-dup) show opposite patterns of white matter (WM) changes compared with healthy controls (HC). Significant macrostructural changes (lower WM/ higher cerebrospinal (CSF) volume) were found in 22q-del but not in 22q-dup. When controlling for macrostructural effects, microstructural changes are no longer significant in 22q-del but remain significant in 22q-dup. Taken together, these results do not support gene dosage as a direct cause for micro- or macrostructural differences in 22q-del and 22q-dup.

While our current analysis cannot deduce causality, we speculate that enlarged CSF volume (i.e., in ventricles and around the brain parenchyma) in 22q-del might contribute to smaller WM volume, which then leads to reduced extracellular space within the WM and therefore more densely packed cellular structures. Several previous studies [97-99] have shown increased CSF in 22q-del, with the most frequent changes observed for the lateral and third ventricle. Further, research utilizing a 22q-del mouse model has reported early and progressive ventricle enlargement due to defective ciliary motility which in turn is caused by elevated dopamine receptor levels [100], suggesting a potential neurobiological mechanism underlying CSF abnormalities in 22q-del. At the same time, postmortem studies in $22 q$-del have suggested that gliotic and neuronal migration defects might alter WM microstructure and density $[25,26]$. We, therefore, acknowledge that future longitudinal studies are needed to determine how micro and macrostructural abnormalities interact with one another in 22q11.2 CNV carriers.
In line with our proposed interpretation of more densely packed WM in 22q-del, other clinical populations that observed higher FA (i.e., infants with extremely preterm birth and individuals with Williams's syndrome) have speculated that the increased FA could be reflective of greater WM packing density [101-103]. Furthermore, diffusion studies in individuals with idiopathic normal pressure hydrocephalus have reported regionally higher FA andin line with our hypothesis-a positive correlation between FA and ventricular volume [104]. We speculate that the neurological consequences of more densely packed WM might include reduced efficiency of electric signal transport or less effective extracellular matrix; however, preclinical studies investigating this possibility in 22q-del models have not yet been done. Indeed, previous research in (mild) traumatic brain injury and neurodegenerative disorders has suggested that axonal swellings or beading translate into disturbed axonal conduction and synaptic transmission [105]. However, we acknowledge that our interpretation is speculative, and future studies are needed to explore the 
underlying neurobiological basis of WM abnormalities in $22 q 11.2$ CNV carriers.

Along the same lines, future longitudinal studies observing macro- and microstructural relationships are needed to explore the role of brain pathologies underlying psychiatric symptoms in $22 q$-del. Similar to our finding of enlarged CSF in 22q-del, previous studies have highlighted the role of increased CSF in various neurological and psychiatric disorders, including dementia [106] and mood disorder [107], and enlarged ventricles are considered one of the hallmarks of psychosis [108]. However, most previous studies have reported decreased rather than increased FA in neuropsychiatric disorders, such as dementia, mood disorders, and psychosis [17-24]. Interestingly, increased FA has been reported in populations at clinical high risk for psychosis, which might be associated with an altered maturational WM trajectory and an earlier peak of WM development [109]. Even though we found increased FA in 22q-del relative to controls, there are important differences from other clinical high-risk populations. Specifically, subjects with $22 q$-del present with various other medical and clinical comorbidities, including autism spectrum disorder, intellectual disability, and anxiety disorder. This, together with the observed widespread macro-and microstructural abnormalities suggests that 22q-del is characterized by early neurodevelopmental abnormalities, which may be independent of the later risk to develop psychosis. Future longitudinal studies, including a follow-up of the current cohort, may shed more light on the relationship between psychosis onset in the 22q-del individuals and changes in dMRI measures.

\section{WM architecture in 22q-dup}

As the first study to investigate WM architecture in 22q-dup, our findings indicate that the 22q11.2 duplication is associated with an opposing pattern of WM alterations to those that characterize 22q-del. Specifically, 22q-dup demonstrated lower $F A$ and $F A_{T}$, lower primary and secondary fiber fractions (F1 and F2), and higher isotropic space (FW and $F_{\text {iso }}$ ) than HC. Interestingly, while we observed a high spatial overlap between abnormalities in 22qdel and 22q-dup, in 22q-dup, microstructural WM abnormalities were independent of macrostructural volume. Similar WM abnormalities (lower $\mathrm{FA} / \mathrm{FA}_{\mathrm{T}}$, higher $\mathrm{FW}$ ) occur in healthy aging, in neurodegenerative disorders such as dementia, as well as psychiatric disorders including depression and schizophrenia [70, 74, 78, 110-112].

The opposing WM architecture findings in 22q-del and 22q-dup are consistent with imaging studies of other reciprocal CNVs, including 15q11.2 and 16p11.2, in which opposite effects on WM structure have also been observed $[113,114]$. Authors of the cited studies speculate that findings might indicate a dosage effect of genes within the affected locus on brain structure. The interpretation of a 22q11.2 gene dosage effect on WM is particularly compelling, given that several genes essential for myelination [115] and cortical circuit formation [116] are located within the $22 q 11.2$ locus. Specifically, the $22 q 11.2$ region encodes the Nogo-66 receptor gene, a growth inhibitor essential for myelin-depended regulation of plasticity [117]. Indeed, one previous study demonstrated that increased FA in 22q-del was associated with an altered dosage of the Nogo-66 receptor gene. The authors speculated that the variations in the Nogo-66 receptor gene might lead to a lack of myelination inhibition, which translates into higher FA [118].

While future studies are needed to further explore the interaction between genes within the 22q11.2 locus and WM structure, our results suggest that the pathologies underlying opposing patterns of WM abnormalities in 22q-del and 22q-dup might differ from one another. Specifically, while lower FA in 22qdup could be related to less myelin, the higher FA observed in $22 q-d e l$ is less likely to result from abnormally increased myelination, given previous postmortem studies $[25,26]$ and the severe functional abnormalities [5, 119] associated with 22q-del. Instead, our findings suggest more complex differences between these reciprocal CNVs, in which microstructural WM changes are related to macrostructural volume changes in 22q11.2 deletion, but not 22q11.2 duplication. Postmortem and animal studies are warranted to determine the underlying neurobiology of the observed effects [120, 121].

\section{Limitations and future directions}

The limited sample size did not allow us to investigate subgroups of 22q11.2 CNV carriers with particular psychiatric phenotypes or to explore the association between brain structure and clinical and cognitive impairments in depth. Future investigations in larger cohorts are warranted to compare 22q-del with and without psychotic symptoms, given the increased risk for psychosis in 22q-del [56]. Specifically, such studies could investigate the relationship of brain structural abnormalities with increased risk for psychosis or other common psychiatric disorders in 22q-del and 22q-dup, such as anxiety, mood, and developmental disorders.

As highlighted above, longitudinal studies are also of interest to establish trajectories of neuroanatomical abnormalities and to further elucidate the complex interaction between microstructural and macrostructural abnormalities. Last, while we applied advanced models to investigate WM pathology in 22q-del and 22q-dup, we could not include all factors that might influence the diffusion signal. For example, we could not account for potential vascular abnormalities, which may be present in 22q11.2 CNV carriers, affecting diffusion MRI measurements [122].

\section{CONCLUSION}

Here, we conducted the first study to apply advanced dMRI analysis methods to elucidate WM microstructure abnormalities in reciprocal 22q11.2 CNVs. Findings revealed opposing effects of the 22q11.2 deletion and duplication on WM architecture. In 22q-del, we identified an association between WM microstructure findings and macrostructural volumetric measures, suggesting microstructural abnormalities are directly related to macrostructural features. WM alterations in 22q-dup, however, appear similar to those observed in other neuropsychiatric disorders such as schizophrenia, bipolar disorder, and dementia, suggesting reduced myelination of WM fibers. Hence, the pathology underlying these opposing effects may differ between 22q-del and 22q-dup and thus does not suggest a direct gene dosage effect on WM. Further longitudinal and translational studies are needed to elucidate the mechanisms underlying WM microstructure and macrostructure alterations resulting from CNV at the $22 q 11.2$ gene locus.

\section{REFERENCES}

1. Owen MJ, Doherty JL. What can we learn from the high rates of schizophrenia in people with 22q11.2 deletion syndrome? World Psychiatry. 2016;15:23-25.

2. Davies EG, Cheung M, Gilmour K, Maimaris J, Curry J, Furmanski A, et al. Thymus transplantation for complete DiGeorge syndrome: European experience. J Allergy Clin Immunol. 2017;140:1660-1670.e1616.

3. Habel A, McGinn MJ 2nd, Zackai EH, Unanue N, McDonald-McGinn DM. Syndrome-specific growth charts for 22q11.2 deletion syndrome in Caucasian children. Am J Med Genet Part A. 2012;158a:2665-2671.

4. Vo OK, McNeill A, Vogt KS. The psychosocial impact of $22 q 11$ deletion syndrome on patients and families: a systematic review. Am J Med Genet Part A. 2018;176:2215-2225.

5. Zhao Y, Guo T, Fiksinski A, Breetvelt E, McDonald-McGinn DM, Crowley TB, et al. Variance of IQ is partially dependent on deletion type among 1,427 22q11.2 deletion syndrome subjects. Am J Med Genet Part A. 2018;176:2172-2181.

6. Vangkilde A, Olsen L, Hoeffding LK, Pedersen CB, Mortensen PB, Werge T, et al. Schizophrenia spectrum disorders in a Danish 22q11.2 Deletion Syndrome Cohort compared to the total Danish population - a Nationwide Register Study. Schizophr Bull. 2016;42:824-831.

7. Olsen L, Sparsø T, Weinsheimer SM, Dos Santos M, Mazin W, Rosengren A, et al. Prevalence of rearrangements in the $22 \mathrm{q} 11.2$ region and population-based risk 
of neuropsychiatric and developmental disorders in a Danish population: a casecohort study. Lancet Psychiatry. 2018;5:573-580.

8. Portnoi MF. Microduplication 22q11.2: a new chromosomal syndrome. Eur J Med Genet. 2009;52:88-93.

9. Portnoï MF, Lebas F, Gruchy N, Ardalan A, Biran-Mucignat V, Malan V, et al. 22q11.2 duplication syndrome: two new familial cases with some overlapping features with DiGeorge/velocardiofacial syndromes. Am J Med Genet Part A. 2005;137:47-51.

10. Agergaard P, Olesen C, Ostergaard JR, Christiansen M, Sorensen KM. Chromosome 22q11.2 duplication is rare in a population-based cohort of Danish children with cardiovascular malformations. Am J Med Genet Part A. 2012;158a:509-513.

11. Mukaddes NM, Herguner S. Autistic disorder and 22q11.2 duplication. World Biol Psychiatry. 2007;8:127-130.

12. Amelsvoort TV, Denayer A, Boermans J, Swillen A. Psychotic disorder associated with 22q11.2 duplication syndrome. Psychiatry Res. 2016;236:206-207.

13. Lo-Castro A, Galasso C, Cerminara C, El-Malhany N, Benedetti S, Nardone AM, et al. Association of syndromic mental retardation and autism with $22 \mathrm{q} 11.2$ duplication. Neuropediatrics. 2009;40:137-140.

14. Brunet A, Armengol L, Pelaez T, Guillamat R, Vallès V, Gabau E, et al. Failure to detect the 22q11.2 duplication syndrome rearrangement among patients with schizophrenia. Behav Brain Funct. 2008;4:10.

15. Rees E, Kirov G, Sanders A, Walters JT, Chambert KD, Shi J, et al. Evidence that duplications of 22q11.2 protect against schizophrenia. Mol Psychiatry. 2014; 19:37-40

16. Malpas CB, Genc S, Saling MM, Velakoulis D, Desmond PM, O'Brien TJ. MRI correlates of general intelligence in neurotypical adults. J Clin Neurosci. 2016;24:128-134.

17. Kelly S, Jahanshad N, Zalesky A, Kochunov P, Agartz I, Alloza C, et al. Widespread white matter microstructural differences in schizophrenia across 4322 individuals: results from the ENIGMA Schizophrenia DTI Working Group. Mol Psychiatry. 2018;23:1261-1269.

18. Favre $P$, Pauling $M$, Stout J, Hozer F, Sarrazin S, Abé $C$, et al. Widespread white matter microstructural abnormalities in bipolar disorder: evidence from megaand meta-analyses across 3033 individuals. Neuropsychopharmacology. 2019;44:2285-2293.

19. Hatton SN, Huynh KH, Bonilha L, Abela E, Alhusaini S, Altmann A, et al. White matter abnormalities across different epilepsy syndromes in adults: an ENIGMAEpilepsy study. Brain. 2020;143:2454-2473.

20. Kamagata K, Andica C, Hatano T, Ogawa T, Takeshige-Amano H, Ogaki $K$, et al. Advanced diffusion magnetic resonance imaging in patients with Alzheimer's and Parkinson's diseases. Neural Regen Res. 2020;15:1590-1600.

21. Koshiyama D, Fukunaga M, Okada N, Morita K, Nemoto K, Usui K, et al. White matter microstructural alterations across four major psychiatric disorders: megaanalysis study in 2937 individuals. Mol Psychiatry. 2020;25:883-895.

22. Sarasso E, Agosta F, Piramide N, Filippi M. Progression of grey and white matter brain damage in Parkinson's disease: a critical review of structural MRI literature. J Neurol. 2021;268:3144-3179.

23. van Velzen LS, Kelly S, Isaev D, Aleman A, Aftanas LI, Bauer J, et al. White matter disturbances in major depressive disorder: a coordinated analysis across 20 international cohorts in the ENIGMA MDD working group. Mol Psychiatry. 2020;25:1511-1525.

24. Piras F, Piras F, Abe $Y$, Agarwal SM, Anticevic A, Ameis $S$, et al. White matter microstructure and its relation to clinical features of obsessive-compulsive disorder: findings from the ENIGMA OCD Working Group. Transl Psychiatry. 2021;11:173.

25. Kiehl TR, Chow EW, Mikulis DJ, George SR, Bassett AS. Neuropathologic features in adults with 22q11.2 deletion syndrome. Cereb Cortex. 2009;19:153-164.

26. Wu P, Teot L, Murdoch G, Monaghan-Nichols AP, McFadden K. Neuropathology of 22q11 deletion syndrome in an infant. Pediatr Dev Pathol. 2014;17:386-392.

27. Padula MC, Scariati E, Schaer M, Eliez S. A mini review on the contribution of the anterior cingulate cortex in the risk of psychosis in $22 \mathrm{q} 11.2$ deletion syndrome. Front Psychiatry. 2018;9:372.

28. Tylee DS, Kikinis Z, Quinn TP, Antshel KM, Fremont W, Tahir MA, et al. Machinelearning classification of 22q11.2 deletion syndrome: a diffusion tensor imaging study. Neuroimage Clin. 2017;15:832-842.

29. Mori S, Zhang J. Principles of diffusion tensor imaging and its applications to basic neuroscience research. Neuron. 2006;51:527-539.

30. Basser PJ, Pierpaoli C. Microstructural and physiological features of tissues elucidated by quantitative-diffusion-tensor MRI. J Magn Reson B. 1996;111:209-219.

31. Assaf $Y$, Pasternak O. Diffusion tensor imaging (DTI)-based white matter mapping in brain research: a review. J Mol Neurosci. 2008;34:51-61.
32. Song SK, Sun SW, Ju WK, Lin SJ, Cross AH, Neufeld AH. Diffusion tensor imaging detects and differentiates axon and myelin degeneration in mouse optic nerve after retinal ischemia. Neuroimage. 2003;20:1714-1722.

33. O'Donnell LJ, Pasternak O. Does diffusion MRI tell us anything about the white matter? An overview of methods and pitfalls. Schizophrenia Res. 2015;161:133-141.

34. Kikinis Z, Makris N, Finn CT, Bouix S, Lucia D, Coleman MJ, et al. Genetic contributions to changes of fiber tracts of ventral visual stream in 22q11.2 deletion syndrome. Brain Imaging Behav. 2013;7:316-325.

35. Deng Y, Goodrich-Hunsaker NJ, Cabaral M, Amaral DG, Buonocore MH, Harvey $D$, et al. Disrupted fornix integrity in children with chromosome 22q11.2 deletion syndrome. Psychiatry Res. 2015;232:106-114.

36. Kates WR, Olszewski AK, Gnirke MH, Kikinis Z, Nelson J, Antshel KM, et al. White matter microstructural abnormalities of the cingulum bundle in youths with 22q11.2 deletion syndrome: associations with medication, neuropsychological function, and prodromal symptoms of psychosis. Schizophr Res. 2015;161:76-84.

37. Jalbrzikowski M, Villalon-Reina JE, Karlsgodt KH, Senturk D, Chow C, Thompson $\mathrm{PM}$, et al. Altered white matter microstructure is associated with social cognition and psychotic symptoms in 22q11.2 microdeletion syndrome. Front Behav Neurosci. 2014;8:393.

38. Nuninga JO, Bohlken MM, Koops S, Fiksinski AM, Mandl R, Breetvelt EJ, et al. White matter abnormalities in 22q11.2 deletion syndrome patients showing cognitive decline. Psychol Med. 2018;48:1655-1663.

39. Olszewski AK, Kikinis Z, Gonzalez CS, Coman IL, Makris N, Gong X, et al. The social brain network in 22q11.2 deletion syndrome: a diffusion tensor imaging study. Behav Brain Funct. 2017;13:4.

40. Dubourg L, Schneider M, Padula MC, Chambaz L, Schaer M, Eliez S. Implication of reward alterations in the expression of negative symptoms in 22q11.2 deletion syndrome: a behavioural and DTI study. Psychol Med. 2017:47:1442-1453.

41. Villalçn-Reina JE, Martjnez K, Qu X, Ching CRK, Nir TM, Kothapalli D. et al. Altered white matter microstructure in 22q11.2 deletion syndrome: a multisite diffusion tensor imaging study. Mol Psychiatry. 2020;25:2818-2831.

42. Lin A, Ching C, Vajdi A, Sun D, Jonas RK, Jalbrzikowski M, et al. Mapping 22q11.2 gene dosage effects on brain morphometry. J Neurosci. 2017;37:6183-6199.

43. Pasternak O, Sochen N, Gur Y, Intrator N, Assaf Y. Free water elimination and mapping from diffusion MRI. Magn Reson Med. 2009;62:717-730.

44. Behrens TE, Berg HJ, Jbabdi S, Rushworth MF, Woolrich MW. Probabilistic diffusion tractography with multiple fibre orientations: what can we gain? Neuroimage. 2007;34:144-155.

45. Jbabdi S, Behrens TE, Smith SM. Crossing fibres in tract-based spatial statistics. Neuroimage. 2010;49:249-256.

46. First, MB, Spitzer, RL, Gibbon, $M$, et al. Structured clinical interview for DSM-IV-TR axis I disorders, research version, non-patient edition (SCID-I/NP). in Biometrics research. New York: NYSP Institute; 2002.

47. Tang KL, Antshel KM, Fremont WP, Kates WR. Behavioral and psychiatric phenotypes in 22q11.2 deletion syndrome. J Dev Behav Pediatrics. 2015;36:639-650.

48. Ousley OY, Smearman E, Fernandez-Carriba S, Rockers KA, Coleman K, Walker $E F$, et al. Axis I psychiatric diagnoses in adolescents and young adults with 22q11 deletion syndrome. Eur Psychiatry. 2013;28:417-422.

49. Schneider $M$, Debbané $M$, Bassett AS, Chow EW, Fung WL, van den Bree $M$, et al. Psychiatric disorders from childhood to adulthood in 22q11.2 deletion syndrome: results from the International Consortium on Brain and Behavior in 22q11.2 Deletion Syndrome. Am J Psychiatry. 2014;171:627-639.

50. Ventura J, Green MF, Shaner A, Liberman RP. Training and quality assurance with the brief psychiatric rating scale: "the drift busters". Int J Methods Psychiatr Res. 1993:3:221-244.

51. Lukoff $\mathrm{D}$, Nuechterlein $\mathrm{KH}$, Ventura J. Manual for the expanded brief psychiatric rating scale. Schizophr Bull. 1986;12:594-602.

52. Lukoff D, Liberman RP, Nuechterlein KH. Symptom monitoring in the rehabilitation of schizophrenic patients. Schizophr Bull. 1986;12:578-602.

53. Miller TJ, McGlashan TH, Rosen JL, Cadenhead K, Cannon T, Ventura J, et al. Prodromal assessment with the Structured Interview for Prodromal Syndromes and the Scale of Prodromal Symptoms: predictive validity, interrater reliability, and training to reliability. Schizophrenia Bull. 2003;29:703-715.

54. Tang SX, Yi JJ, Moore TM, Calkins ME, Kohler CG, Whinna DA, et al. Subthreshold psychotic symptoms in 22q11.2 deletion syndrome. J Am Acad Child Adolesc Psychiatry. 2014;53:991-1000.e1002.

55. Zhan L, Jenkins LM, Zhang A, Conte G, Forbes A, Harvey D, et al. Baseline connectome modular abnormalities in the childhood phase of a longitudinal study on individuals with chromosome 22q11.2 deletion syndrome. Hum Brain Mapp. 2018;39:232-248. 
56. Monks S, Niarchou M, Davies AR, Walters JT, Williams N, Owen MJ, et al. Further evidence for high rates of schizophrenia in 22q11.2 deletion syndrome. Schizophr Res. 2014;153:231-236.

57. Diagnostic And Statistical Manual of Mental Disorders: DSM-IV (Washington, DC, 1994)

58. Wilkinson, G. The wide range achievement test-revision 3 (Jastak Association, Wilmington, 1993).

59. Wilkinson, GS \& Robertson, GJ. WRAT 3 administration manual (Jastak Associates, Wilmington, DE, 1993).

60. Glasser MF, Sotiropoulos SN, Wilson JA, Coalson TS, Fischl B, Andersson JL, et al. The minimal preprocessing pipelines for the Human Connectome Project. Neuroimage. 2013;80:105-124.

61. Andersson JL, Skare S, Ashburner J. How to correct susceptibility distortions in spin-echo echo-planar images: application to diffusion tensor imaging. Neuroimage. 2003;20:870-888.

62. Andersson JLR, Sotiropoulos SN. An integrated approach to correction for offresonance effects and subject movement in diffusion MR imaging. Neuroimage. 2016;125:1063-1078.

63. Koay CG, Chang LC, Carew JD, Pierpaoli C, Basser PJ. A unifying theoretical and algorithmic framework for least squares methods of estimation in diffusion tensor imaging. J Magn Reson. 2006;182:115-125.

64. Pasternak O, Shenton ME, Westin CF. Estimation of extracellular volume from regularized multi-shell diffusion MRI. Med Image Comput Comput Assist Interv. 2012;15:305-312.

65. Hoy AR, Koay CG, Kecskemeti SR, Alexander AL. Optimization of a free water elimination two-compartment model for diffusion tensor imaging. Neuroimage. 2014;103:323-333.

66. Metzler-Baddeley C, O'Sullivan MJ, Bells S, Pasternak O, Jones DK. How and how not to correct for CSF-contamination in diffusion MRI. Neuroimage. 2012;59:1394-1403.

67. Di Biase MA, Zalesky A, Cetin-Karayumak S, Rathi Y, Lv J, Boerrigter D. LargeScale Evidence for an Association Between Peripheral Inflammation and White Matter Free Water in Schizophrenia and Healthy Individuals. Schizophr Bull. 2021;47:542-542.

68. Lyall AE, Pasternak O, Robinson DG, Newell D, Trampush JW, Gallego JA, et al. Greater extracellular free-water in first-episode psychosis predicts better neurocognitive functioning. Mol Psychiatry. 2018;23:701-707.

69. Bergamino M, Kuplicki R, Victor TA, Cha YH, Paulus MP. Comparison of two different analysis approaches for DTI free-water corrected and uncorrected maps in the study of white matter microstructural integrity in individuals with depression. Hum Brain Mapp. 2017;38:4690-4702.

70. Tuozzo C, Lyall AE, Pasternak O, James A, Crow TJ, Kubicki M. Patients with chronic bipolar disorder exhibit widespread increases in extracellular free water. Bipolar Disord. 2018;20:523-530.

71. Breithaupt L, Chunga-Iturry N, Lyall AE, Cetin-Karayumak S, Becker KR, Thomas $\mathrm{JJ}$, et al. Developmental stage-dependent relationships between ghrelin levels and hippocampal white matter connections in low-weight anorexia nervosa and atypical anorexia nervosa. Psychoneuroendocrinology. 2020;119:104722.

72. Pasternak O, Koerte IK, Bouix S, Fredman E, Sasaki T, Mayinger M, et al. Hockey Concussion Education Project, Part 2. Microstructural white matter alterations in acutely concussed ice hockey players: a longitudinal free-water MRI study. J Neurosurg. 2014;120:873-881.

73. Maier-Hein KH, Westin CF, Shenton ME, Weiner MW, Raj A, Thomann P, et al. Widespread white matter degeneration preceding the onset of dementia. Alzheimers Dement. 2015;11:485-493.e482.

74. Hoy AR, Ly M, Carlsson CM, Okonkwo OC, Zetterberg H, Blennow K, et al. Microstructural white matter alterations in preclinical Alzheimer's disease detected using free water elimination diffusion tensor imaging. PLoS ONE. 2017;12:e0173982.

75. Guttuso T Jr, Bergsland N, Hagemeier J, Lichter DG, Pasternak O, Zivadinov R Jr. Substantia nigra free water increases longitudinally in Parkinson Disease. AJNR Am J Neuroradiol. 2018:39:479-484.

76. Planetta PJ, Ofori E, Pasternak O, Burciu RG, Shukla P, DeSimone JC, et al. Freewater imaging in Parkinson's disease and atypical parkinsonism. Brain. 2016;139:495-508.

77. Pasternak O, Kubicki M, Shenton ME. In vivo imaging of neuroinflammation in schizophrenia. Schizophr Res. 2016;173:200-212.

78. Chad JA, Pasternak O, Salat DH, Chen JJ. Re-examining age-related differences in white matter microstructure with free-water corrected diffusion tensor imaging. Neurobiol Aging. 2018;71:161-170.

79. Douaud G, Jbabdi S, Behrens TE, Menke RA, Gass A, Monsch AU, et al. DTI measures in crossing-fibre areas: increased diffusion anisotropy reveals early white matter alteration in $\mathrm{MCl}$ and mild Alzheimer's disease. Neuroimage. 2011;55:880-890.
80. Smith SM, Jenkinson M, Johansen-Berg H, Rueckert D, Nichols TE, Mackay CE, et al. Tract-based spatial statistics: voxelwise analysis of multi-subject diffusion data. Neuroimage. 2006;31:1487-1505

81. Winkler AM, Ridgway GR, Webster MA, Smith SM, Nichols TE. Permutation inference for the general linear model. Neuroimage. 2014;92:381-397.

82. Smith SM, Nichols TE. Threshold-free cluster enhancement: addressing problems of smoothing, threshold dependence and localisation in cluster inference. Neuroimage. 2009;44:83-98.

83. Avants BB, Tustison NJ, Song G, Cook PA, Klein A, Gee JC. A reproducible evaluation of ANTs similarity metric performance in brain image registration. Neuroimage. 2011;54:2033-2044.

84. Keihaninejad S, Ryan NS, Malone IB, Modat M, Cash D, Ridgway GR, et al. The importance of group-wise registration in tract based spatial statistics study of neurodegeneration: a simulation study in Alzheimer's disease. PLoS ONE. 2012;7:e45996.

85. Bach M, Laun FB, Leemans A, Tax CM, Biessels GJ, Stieltjes B, et al. Methodological considerations on tract-based spatial statistics (TBSS). Neuroimage. 2014;100:358-369.

86. Jeurissen B, Leemans A, Tournier JD, Jones DK, Sijbers J. Investigating the prevalence of complex fiber configurations in white matter tissue with diffusion magnetic resonance imaging. Hum Brain Mapp. 2013;34:2747-2766.

87. Zhang F, Breger A, Ning L, Westin CF, O'Donnell L, Pasternak O. Deep learning based brain tissue segmentation from novel diffusion kurtosis imaging features. Annual Meeting of the International Society for Magnetic Resonance in Medicine, 2020

88. Seitz J, Sawyer KS, Papadimitriou G, Oscar-Berman M, Ng I, Kubicki A, et al. Alcoholism and sexual dimorphism in the middle longitudinal fascicle: a pilot study. Brain Imaging Behav. 2017;11:1006-1017.

89. Seitz J, Lyall AE, Kanayama G, Makris N, Hudson Jl, Kubicki M, et al. White matter abnormalities in long-term anabolic-androgenic steroid users: a pilot study. Psychiatry Res Neuroimaging. 2017;260:1-5

90. Kubicki M, Westin C-F, McCarley RW, Shenton ME. The Application of DTI to Investigate White Matter Abnormalities in Schizophrenia. Ann N Y Acad Sci. 2005;1064:134-148.

91. Sagarwala R, Nasrallah HA. A systematic review of diffusion tensor imaging studies in drug-naive OCD patients before and after pharmacotherapy. Ann Clin Psychiatry. 2020;32:42-47.

92. Dennis EL, Wilde EA, Newsome MR, Scheibel RS, Troyanskaya M, Velez C, et al. Enigma military brain injury: a coordinated meta-analysis of diffusion mri from multiple cohorts. Proc IEEE Int Symp Biomed Imaging. 2018;2018:1386-1389.

93. Barona M, Brown M, Clark C, Frangou S, White T, Micali N. White matter alterations in anorexia nervosa: evidence from a voxel-based meta-analysis. Neurosci Biobehav Rev. 2019;100:285-295.

94. Barnea-Goraly N, Menon V, Krasnow B, Ko A, Reiss A, Eliez S. Investigation of white matter structure in velocardiofacial syndrome: a diffusion tensor imaging study. Am J Psychiatry. 2003:160:1863-1869.

95. Roalf DR, Eric Schmitt J, Vandekar SN, Satterthwaite TD, Shinohara RT, Ruparel K, et al. White matter microstructural deficits in 22q11.2 deletion syndrome. Psychiatry Res Neuroimaging. 2017;268:35-44.

96. Kikinis Z, Cho K, Coman IL, Radoeva PD, Bouix S, Tang Y, et al. Abnormalities in brain white matter in adolescents with 22q11.2 deletion syndrome and psychotic symptoms. Brain Imaging Behav. 2017;11:1353-1364.

97. Eliez S, Schmitt JE, White CD, Reiss AL. Children and adolescents with velocardiofacial syndrome: a volumetric MRI study. Am J Psychiatry. 2000;157:409-415.

98. Baker K, Chaddock CA, Baldeweg T, Skuse D. Neuroanatomy in adolescents and young adults with $22 \mathrm{q} 11$ deletion syndrome: comparison to an IQ-matched group. Neuroimage. 2011;55:491-499.

99. Forrester S, Kovach MJ, Smith RE, Rimer L, Wesson M, Kimonis VE. Kousseff syndrome caused by deletion of chromosome 22q11-13. Am J Med Genet. 2002;112:338-342.

100. Eom TY, Han SB, Kim J, Blundon JA, Wang YD, Yu J, et al. Schizophrenia-related microdeletion causes defective ciliary motility and brain ventricle enlargement via microRNA-dependent mechanisms in mice. Nat Commun. 2020;11:912.

101. Haas BW, Barnea-Goraly N, Sheau KE, Yamagata B, Ullas S, Reiss AL. Altered microstructure within social-cognitive brain networks during childhood in Williams syndrome. Cereb Cortex. 2014;24:2796-2806.

102. Hoeft F, Barnea-Goraly N, Haas BW, Golarai G, Ng D, Mills D, et al. More is not always better: increased fractional anisotropy of superior longitudinal fasciculus associated with poor visuospatial abilities in Williams syndrome. J Neurosci. 2007:27:11960-11965.

103. Rogers CE, Smyser T, Smyser CD, Shimony J, Inder TE, Neil JJ. Regional white matter development in very preterm infants: perinatal predictors and early developmental outcomes. Pediatr Res. 2016;79:87-95. 
104. Siasios I, Kapsalaki EZ, Fountas KN, Fotiadou A, Dorsch A, Vakharia K, et al. The role of diffusion tensor imaging and fractional anisotropy in the evaluation of patients with idiopathic normal pressure hydrocephalus: a literature review. Neurosurg Focus. 2016;41:E12.

105. Gu C. Rapid and reversible development of axonal varicosities: a new form of neural plasticity. Front Mol Neurosci. 2021;14:610857.

106. Nestor SM, Rupsingh R, Borrie M, Smith M, Accomazzi V, Wells JL, et al. Ventricular enlargement as a possible measure of Alzheimer's disease progression validated using the Alzheimer's disease neuroimaging initiative database. Brain. 2008;131:2443-2454.

107. Kempton MJ, Geddes JR, Ettinger U, Williams SC, Grasby PM. Meta-analysis, database, and meta-regression of 98 structural imaging studies in bipolar disorder. Arch Gen Psychiatry. 2008;65:1017-1032.

108. Shenton ME, Dickey CC, Frumin M, McCarley RW. A review of MRI findings in schizophrenia. Schizophr Res. 2001;49:1-52.

109. Di Biase MA, Cetin-Karayumak S, Lyall AE, Zalesky A, Cho KIK, Zhang F, et al. White matter changes in psychosis risk relate to development and are not impacted by the transition to psychosis. Mol Psychiatry (2021). Online ahead of print.

110. Bergamino M, Pasternak O, Farmer M, Shenton ME, Hamilton JP. Applying a free-water correction to diffusion imaging data uncovers stress-related neural pathology in depression. Neuroimage Clin. 2016;10:336-342.

111. Pasternak O, Westin CF, Bouix S, Seidman LJ, Goldstein JM, Woo TU, et al. Excessive extracellular volume reveals a neurodegenerative pattern in schizophrenia onset. J Neurosci. 2012;32:17365-17372.

112. Oestreich LK, Pasternak O, Shenton ME, Kubicki M, Gong X, Australian Schizophrenia Research $B$, et al. Abnormal white matter microstructure and increased extracellular free-water in the cingulum bundle associated with delusions in chronic schizophrenia. Neuroimage Clin. 2016;12:405-414.

113. Chang YS, Owen JP, Pojman NJ, Thieu T, Bukshpun P, Wakahiro ML, et al. Reciprocal white matter alterations due to $16 \mathrm{p} 11.2$ chromosomal deletions versus duplications. Hum Brain Mapp. 2016;37:2833-2848.

114. Silva Al, Ulfarsson MO, Stefansson H, Gustafsson O, Walters GB, Linden D, et al. Reciprocal white matter changes associated with copy number variation at 15q11.2 BP1-BP2: a diffusion tensor imaging study. Biol Psychiatry. 2019;85:563-572.

115. Jungerius BJ, Hoogendoorn ML, Bakker SC, Van't Slot R, Bardoel AF, Ophoff $\mathrm{RA}$, et al. An association screen of myelin-related genes implicates the chromosome 22q11 PIK4CA gene in schizophrenia. Mol Psychiatry. 2008;13: 1060-1068.

116. Meechan DW, Maynard TM, Tucker ES, LaMantia AS. Three phases of DiGeorge/ 22q11 deletion syndrome pathogenesis during brain development: patterning, proliferation, and mitochondrial functions of 22q11 genes. Int J Dev Neurosci. 2011;29:283-294.

117. Budel S, Padukkavidana T, Liu BP, Feng Z, Hu F, Johnson S, et al. Genetic variants of Nogo-66 receptor with possible association to schizophrenia block myelin inhibition of axon growth. J Neurosci. 2008;28:13161-13172.

118. Perlstein MD, Chohan MR, Coman IL, Antshel KM, Fremont WP, Gnirke MH, et al. White matter abnormalities in 22q11.2 deletion syndrome: preliminary associations with the Nogo-66 receptor gene and symptoms of psychosis. Schizophr Res. 2014;152:117-123.

119. Duijff SN, Klaassen PW, de Veye HF, Beemer FA, Sinnema G, Vorstman JA Cognitive development in children with 22q11.2 deletion syndrome. Br J Psychiatry. 2012;200:462-468.

120. Meechan DW, Maynard TM, Tucker ES, Fernandez A, Karpinski BA, Rothblat LA, et al. Modeling a model: mouse genetics, 22q11.2 Deletion Syndrome, and disorders of cortical circuit development. Prog Neurobiol. 2015;130:1-28.
121. Piskorowski RA, Nasrallah K, Diamantopoulou A, Mukai J, Hassan SI, Siegelbaum $\mathrm{SA}$, et al. Age-dependent specific changes in area CA2 of the hippocampus and social memory deficit in a mouse model of the $22 q 11.2$ deletion syndrome. Neuron. 2016;89:163-176.

122. Rydhög AS, Szczepankiewicz F, Wirestam R, Ahlgren A, Westin CF, Knutsson L, et al. Separating blood and water: perfusion and free water elimination from diffusion MRI in the human brain. Neuroimage. 2017;156:423-434.

\section{ACKNOWLEDGEMENTS}

This work was supported by the National Institute of Mental Health R01MH085953, R01MH108574, 1R21MH116473-01A1, and 1U01MH119736.

\section{AUTHOR CONTRIBUTIONS}

$\mathrm{CEB}, \mathrm{OP}$, and JSH designed the study. $\mathrm{LK}, \mathrm{AL}$, and JEV-R were involved in data collection. JSH, ML, KIKC, FZ, and TB conducted data analyses. JSH, ML, LK, AL, JEV-R, $\mathrm{KIKC}, \mathrm{FZ}, \mathrm{SB}, \mathrm{MK}, \mathrm{CEB}$, and OP interpreted the data. JSH, OP, and CEP drafted the article and all authors contributed to revisions and approval of the final draft.

\section{COMPETING INTERESTS}

The authors declare no competing interests.

\section{ADDITIONAL INFORMATION}

Supplementary information The online version contains supplementary material available at https://doi.org/10.1038/s41398-021-01703-1.

Correspondence and requests for materials should be addressed to Johanna SeitzHolland.

Reprints and permission information is available at http://www.nature.com/ reprints

Publisher's note Springer Nature remains neutral with regard to jurisdictional claims in published maps and institutional affiliations.

(i) Open Access This article is licensed under a Creative Common Attribution 4.0 International License, which permits use, sharing, adaptation, distribution and reproduction in any medium or format, as long as you give appropriate credit to the original author(s) and the source, provide a link to the Creative Commons license, and indicate if changes were made. The images or other third party material in this article are included in the article's Creative Commons license, unless indicated otherwise in a credit line to the material. If material is not included in the article's Creative Commons license and your intended use is not permitted by statutory regulation or exceeds the permitted use, you will need to obtain permission directly from the copyright holder. To view a copy of this license, visit http://creativecommons. org/licenses/by/4.0/.

(c) The Author(s) 2021 